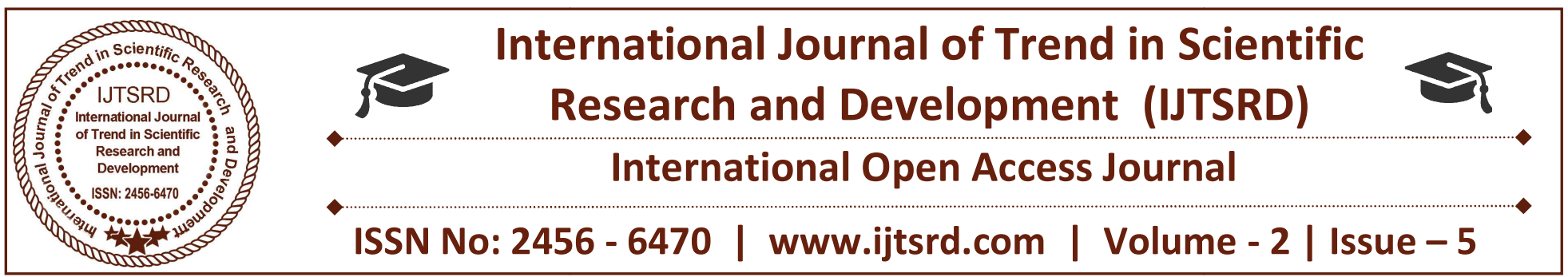

\title{
Photothermal Boiling in Aqueous Nanofluids
}

\author{
D. Navilan \\ Lecturer (Selection Grade), Department of Physics, \\ Sankar Polytechnic College, Tirunelveli, Tamil Nadu, India
}

\begin{abstract}
The process of photo thermal evaporation in nanofluids finds promising applications in solar energetics, medicine and process technology. Previous studies report highly efficient production of solar steam in fluids with gold nanoparticles. In this article, we establish the process in nanofluids with less expensive carbon black (CB) and iron oxide (IO) nanoparticles (NP). Screening the concentration of nanoparticles, we tailor the nanofluids to reach the efficiency maxima: $66 \%$ at $3 \% \mathrm{wt}$. (CBNP) and $75 \%$ at $10 \%$ wt (IONP); the steam was superheated up to $10 \mathrm{~K}$ (CBNP) and $16 \mathrm{~K}$ (IONP). It was also discovered that the IONPs-generated steam was contaminated with nanoparticles. In addition to experimental results, we have developed an empirical model of photo-thermal steam generation in nanofluids. The model agrees well with the experiments.
\end{abstract}

KEYWORD: solar steam, nanofluid, boiling, carbon black, iron oxide

\section{INTRODUCTION}

When nanofluid (NF) (i.e. fluid with suspended nanoparticles) is exposed to thermal radiation, the nanoparticles (NPs) effectively absorb the heat and transmit it into the surrounding fluid. In this case the inter-phase heat transfer becomes intensive because the integral nanoparticle-fluid contact area is sufficiently greater (by at least two orders) than in a traditional solar collector, where a continuous surface absorbs the sunlight. The micro- scale convection around the NPs and surface Plasmon resonance at metallic NPs additionally intensifies the heat transfer. The nanofluids are thus used as coolants in modern direct absorption solar collectors (DASC). Otanicar et al. [1] reported enhancement of the collector efficiency by $7 \%$ when $0.25 \%$ wt of 20 -nm silver NPs was dispersed in water in comparison with a "black surface" absorber. Karami et al. [2] succeeded in improving the photothermal performance of their solar collector by $25 \%$ using 20 -nm copper oxide NPs at $0.01 \% \mathrm{wt}$.

According to the most recent experimental observations and theoretical estimates [3, 4], DASCs accumulate heat most effectively at around $0.50 \% \mathrm{wt}$ NPs.

Although the nanofluid-assisted DASC technology is presently under evolutionary development aiming at a better stability of the nanofluids and screening new types of NPs, this method is rather well established. Nevertheless, another DASC application of the nanofluids that is attracting increased interest and is presently less well-known is the process of solar steam generation. Here the nanoparticles heat the carrier fluid up to saturation and generate steam that potentially can be used in micro-CHP systems, solar distillation and desalination. Other potential applications of the process include nanography, target cancer therapy, solar pond technology and selective evaporation.

The first experimental record on in-situ photothermal evaporation of nanofluid came from the Rice University [5]. By placing an aqueous suspension of $120-170 \mathrm{~nm} \mathrm{SiO} 2 \mathrm{NPs}$, covered with gold shells, under the concentrated sunlight ( $>$ X100), the authors initiated subcooled boiling of the nanofluid with $82 \%$ efficiency. A subsequent experimental work published by the same research group [6] reports a pilot industrial test of the process. The transparent photothermal reactor with the nanofluid (NP number 
density $1010 \mathrm{~m}-3$ ) was located in the focus of the solar dish collector. The solar steam produced in the reactor was used for sterilization of waste by superheating up to $35 \mathrm{~K}$, i.e. this steam could be used to drive a turbine. This concept was also adopted for the process of nanoparticle-driven photo-distillation [7] and off-grid bioethanol production [8].

From the provided literature survey, we conclude that the following issues remain unclear:

1. The composition of the nanofluid that results with the most efficient steam generation;

2. The degree of the solar steam contamination with nanoparticles; (iii) a theoretical description of the process. The present contribution comes with a multi-factor examination of two different nanofluids from the photothermal evaporation point of view. Looking for a cost-effective solution, we produced nanofluids by use of tap water and widely available commercial nanopowders: carbon black (CB) and iron oxide (IO). At first, we aimed to de- fine the optimum NP concentration of the nanofluids, focusing on superheat and efficiency of the process. Next, we condensed the generated steam and inspected NPcontamination of the condensate. Finally, we modified an existing theory of photothermal boiling to connect the kinetics of steam generation to the granulometry of the nanofluid and to reproduce the experimental results theoretically.

\section{EXPERIMENTS}

\subsection{Photo-thermal experiments}

The experimental set-up, schematically illustrated in Fig. 2 (left), consisted of: a cylindrical glass tube (ID $13.5 \mathrm{~mm}$, height $148 \mathrm{~mm}$ ), sealed from the top with a rubber plug; two halogen lamps OSRAM (Haloline $230 \mathrm{~V} 400 \mathrm{~W}$ ), radiating at the glass tube; a 200-ml cylindrical condensate collector and a spiral, flexible PVC piping (ID $3 \mathrm{~mm}$, length $48 \mathrm{~cm}$ ) which was connected the glass tube to the collector. The steam, produced in the glass tube, was completely condensed in the PVC-pipe due to the natural convection of the surrounding air at ambient conditions $\left(23-25^{\circ} \mathrm{C}, 1\right.$ bar).

The two lamps were placed perpendicularly (Fig. 2) in order to prevent mutual heatingof the lamps. The glass tube was located at the $10-\mathrm{cm}$ distance from each lamp, corresponding to the radiate heat flux of $5760 \mathrm{~W} / \mathrm{m} 2$ from a single lamp. The sample was positioned outside the convective zone, which was formed in the air around the lamps [22]. The ther-mal radiation intensity from a single lamp at different distances along the light-path was measured with LS122 radiometer from Aermanda. The radiometer was calibrated in-house using Sol3A Class AAA Solar Simulator from AZPECT. This information is provided in the supplementary material (Fig. S.2). The electromagnetic spectrum of the lamps, determined by the use of Ramses AAC-VIS radiometer ( $0.2 \mathrm{~nm}$, TriOS), was shifted $350 \mathrm{~nm}$ to the red zone relative to the solar spectrum; this was also confirmed by OSRAM [23].

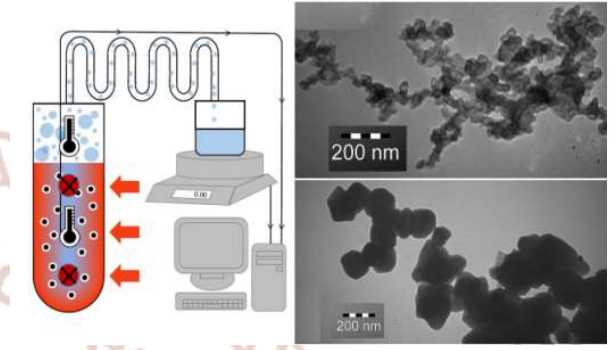

Figure 1: Schematic description of experiments (left), TEM images of NPs (right)

The measurement system included two T-type thermocouples from Omega $\left(0.3^{\circ}\right)$, located along the centerline of the glass tube at 2.2 and $13.1 \mathrm{~cm}$ from the top in order to continuously immerse the sensors into the steam and the fluid phase. The first sensor (located in the steam) was placed outside the irradiated zone. The distance between the lamps and the second sensor $(10.7 \mathrm{~cm})$ was greater than the optical depth of the nanofluids. The measurement was thereby not significantly influenced by thermal radiation of the lamps. The condensate collection rate was registered with the precision scale Sartorius CPA $324 \mathrm{~S}(0.1 \mathrm{mg})$. The scale and the thermocouples (via the digital transmitter) were connected to a PC, recording the measurement every second. During the experiments we altered mass fraction of CBNPs and IONPs in a 5-ml nanofluid sample up to $10 \%$ wt. Higher concentrations are not considered in the present paper since the nanofluids became unstable and formed a stationary viscous bed at the bottom of the sample above this limit. The evap- oration of each sample was performed for 30 minutes. The presence of nanoparticles in the condensate, collected after each run, was inspected by means of Raman spectrometry (RamanRxn1 Analyzer) and static light scattering (Fritsch Analysette 22).

\section{RESULTS AND DISCUSSION}

Boiling of both nanofluids started after a short heating period ( $7 \mathrm{~min}$, a similar heating time was reported by 
[4]), during which the nanofluid temperature rose from the ambient temperature up to the saturation conditions (see Figs. S. 3. 1-S. 3. 2) almost independently on particle concentration. After this point, IONF temperature became constant until the end of each irradiation session. For this nanofluid, no steam with significant superheat was produced in the bulk of the fluid. CBNF nanofluid boiled at low superheat values $(2-3 \mathrm{~K})$ for the samples with concentration below $1 \%$. CBNF temperature for higher concentrations did not differ from IONF.
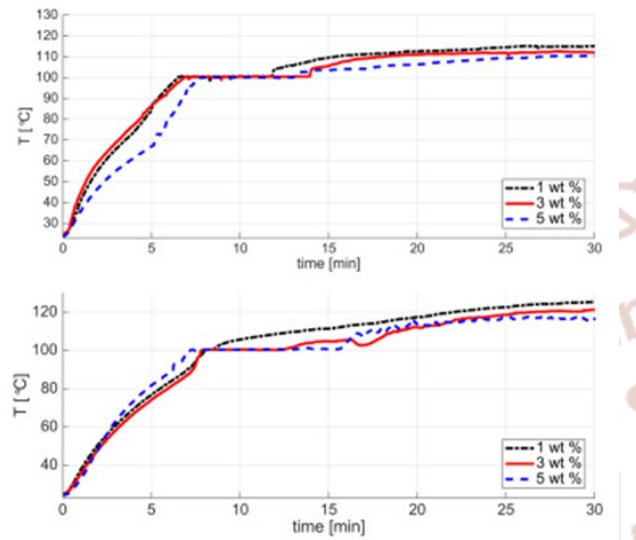

Figure 3: Temporal history of steam temperature for CBNF (top) and IONF (bottom) at particle concentrations $1 \%, 3 \%$ and $5 \%$.

The temperature, detected by the steam sensor, is shown in Fig. 3 for both nanofluids. At the initial steps of the process CBNF steam temperature did not deviate significantly from the nanofluid temperature. After the saturation conditions are established, the temperature remains at saturation until $20 \%$ of the sample has evaporated. IONF did not exhibit any sufficient temporal delay in saturation at particle fractions below $2 \% \mathrm{wt}$, even though it demonstrated behavior similar to CBNF at larger concentrations. The magnitude of the superheat was almost twice greater for IONF steam. This is demonstrated in the figure and Table 1, which shows time-average superheat for both nanofluids at different concentrations.

\begin{tabular}{|c|c|c|}
\hline Concentration & CBNF & IONF \\
\hline 0.3 & 5.0 & 13.1 \\
\hline 0.5 & 7.4 & 14.4 \\
\hline 1.0 & 9.5 & 15.8 \\
\hline 2.0 & 8.3 & 13.6 \\
\hline 3.0 & 6.0 & 10.1 \\
\hline 4.0 & 6.2 & 6.1 \\
\hline 5.0 & 6.9 & 9.1 \\
\hline 10.0 & 6.7 & 11.3 \\
\hline
\end{tabular}

Table 1: Time-average superheat $\Delta \mathrm{T}(\mathrm{K})$ for different NP concentration
CBNF steam increases superheating with a concentration up to the maximum of $9.5 \mathrm{~K}$ at $1 \%$. Further, reduction of superheat is weakly dependent on concentration. To our knowledge, there has been no equivalent superheat detected in previous studies of CBNF under external radiation. Moreover, these values exceeded $6.5 \mathrm{~K}$ that corresponds to conventional water boiling curve at $5760 \mathrm{~W} / \mathrm{m} 2$ [24] from a heating surface under normal conditions. According to our supplementary computational fluid dynamics (CFD) analysis, performed for the steam flow in the experimental set-up (Fig. S.4), the steam pressure was negligibly over 1 bar so that the superheat cannot be explained by over-pressurization. The superheat of IONF steam is dependent on the nanoparticle concentration in an equivalent way, having a maximum of $15.8 \mathrm{~K}$ at $1 \%$. Amjad et al. [25] and Neumann et al. $[5,6]$ observed similar values of superheat for the Au-water system heated with 200 sun.

Figure 4 presents evaporation kinetics for both nanofluids. Both nanofluids produced steam with the mass flow of similar magnitude, clearly demonstrating of an optimum con- cent ration between $1 \%$ wt and $5 \%$ wt, above which the evaporation drops down. When considering these results, it is possible to denote two opposite drivers that define qualitative behavior of the steam temperature: more intensive trapping of the nanoparticle thermal boundary layers ( $\mathrm{Ni}$ et al. [4]) due to evaporation (and respective densification of the particulate phase) is responsible for increasing superheat with time; the reduction of superheat with particle concentration is caused by more intensive absorption of the light at the boundaries of the experimental system and so limited exposure of the bulk. Taking into account density and size difference between CBNPs and IONPs, it is important to note that IONF generates steam more effectively even ППattributed to multiple differences between the mentioned nanofluids: IONF is of higher thermal conductivity [26] and is better exposed by thermal radiation in the bulk; IONPs are hydrophilic and surface Plasmon resonance takes place at the IONPs. Moreover, the difference in the nanofluid granulometry that influences boiling kinetics must be taken into account and is discussed in the following section. 


\subsection{Efficiencies}

Another crucial technical parameter that describes the overall thermal performance of the considered system is process efficiency. Here, depending on application, two possible cases were considered. The heat absorption efficiency, which is important if the nanofluid is planned to be exploited in a direct absorption solar collector without boiling:

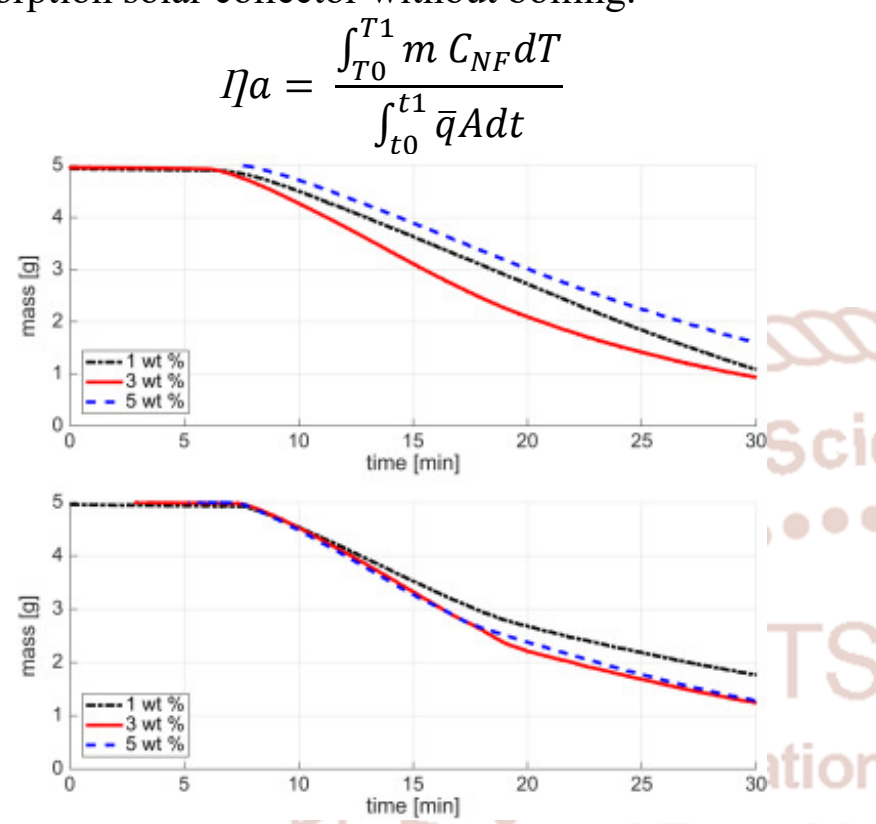

Figure 4: Mass of the sample as a function of time for

CBNF (top) and IONF (bottom) at particle concentrations $1 \%, 3 \%$ and $5 \%$.

Where CNF is the specific heat, $\mathrm{m}$ is the mass of the nanofluid and $\mathrm{A}$ is the irradiated area of the collector; these variables are time-dependent. Equation (1) is to be integrated from the start of the experiment, $t=t 0$, until the boiling point has been reached at the time $t 1$. This corresponds to the initial temperature at $\mathrm{T} 0$ and the saturation temperature Ts. The specific heat CNF is estimated assuming homogeneous mixture of the fluid and the nanoparticles, scaling the respective parameters of each phase with the time-specific mass fraction of the nanoparticles $\mathrm{fNP}: \mathrm{CNF}=\mathrm{Cl}(1 \mathrm{fNP})$ + CNP fNP (indices NP and 1 denote the nanoparticles and the base liquid). The average heat flux from the lamps $q$ is defined as $q=(2 / D) D q(z) d z$, where $z$ is the direction of lamp light, $\mathrm{D}$ is the internal diameter of the experimental tube (Fig. 2) and $q(z)$ is a fitted function of experimental measurement.

The time-average absorption efficiency for our experimental system is plotted against concentration in Fig. 5. It follows from the figure that the efficiency was always above $55.0 \%$, increasing with the particle concentration up to $66.7 \%$ at the optimum concentration of $1 \% \mathrm{wt}$ for CBNF. This concentration was twice greater than the one reported in [3] and [4] as the optimum of photo thermal absorption. After this point the efficiency starts to decay with CBNPs concentration, while the photo thermal performance of IONF increases continuously with concentration up to $84.2 \%$. The qualitative dependence of absorption efficiency on particle concentration is equivalent to the one discussed for superheat (Table 1). There is however an alternative explanation for the existence optimum concentration by Mynkowycz et al. [26], who suggested that the most intensive natural convection established in the nanofluid with the maximum achievable effective thermal conductivity and the minimum possible effective viscosity. Both parameters, being proportional to the concentration of nanoparticles, rich equilibrium at volume fractions which correspond to $2-5 \%$ wt. These values differ from the optimum, detected in our study.

It is interesting to compare the photo thermal performance of our system to the results reported in other relevant works, which are also shown in Fig. 5. Here we conclude that a number of different nanofluids exhibited similar photo thermal performance, increasing the efficiency up to the optimum $\mathrm{fNP}<1 \%$ and slightly reducing the photo thermal performance above this concentration. Considering points (3) and (4) from Fig. 5 for $\mathrm{Ag}$ nanofluid of the same concentration but different particle size [1], it is possible to conclude that the photo thermal performance is inversely proportional to the size of the nanoparticles. The absorption efficiency of most of the third-party systems was however, slightly lower than in our case even though much smaller nanoparticles were used (up to $50 \mathrm{~nm}$ ). This observation may be explained by the absence of fully developed natural convection of the nanofluid during the experiment. Indeed, a major part of these systems were heated by a lower radiative heat flux (typically 1 sun), coming from the top of the solar collector, i.e. the convective currents of expectantly lower magnitude could establish there only due to sedimentation and thermophoresis of nanoparticles. Moreover, as it follows from point (2) in the figure, the photothermal performance of the system does not sufficiently improve when the radiative heat flux approached our values (10 sun) while still being directed along the direction of gravity. The importance of convective mass transfer in the bulk of the fluid is further illustrated by point (1) where the recirculating solar collector system was considered. Here the nanofluid was mixed due to the forced 
convection that resulted with the absorption efficiency well above $80 \%$ at the mass concentration of nanoparticles below $0.1 \%$.

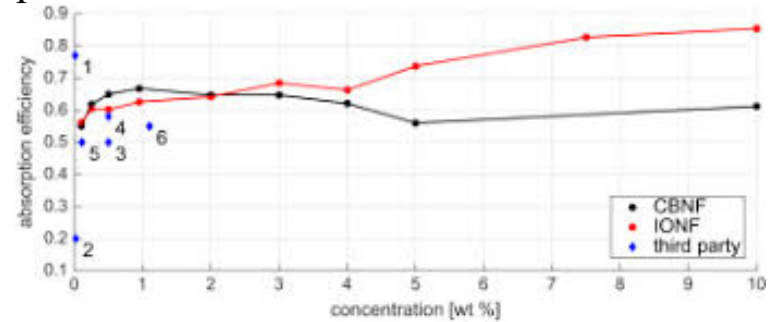

Figure 5: Time-average absorption efficiency as a function of nanoparticle concentration for CBNF and IONF

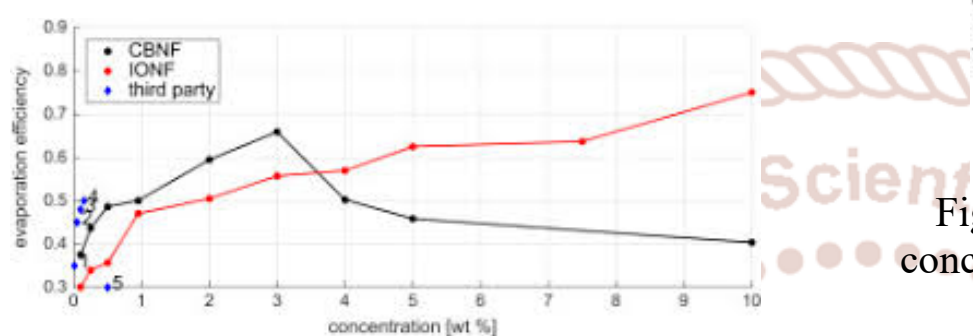

Figure 7: Steam production as a function of mass concentration. CBNF and IONF results are compared $\checkmark$ with Equation

requires about $300 \mathrm{l} / \mathrm{min}$. This would correspond to the production of steam out of our NFs in a solar concentrator with irradiated area up to $1 \mathrm{~m} 2$. This generation would potentially result in 500 we without steam overpressure. Accounting for 11-fold magnification, which is equivalent to our experiments, the target steam generation rate requires up to $11 \mathrm{~m} 2$ of solar concentrator, which is obviously a lowest estimate and does not account for possible thermal losses.

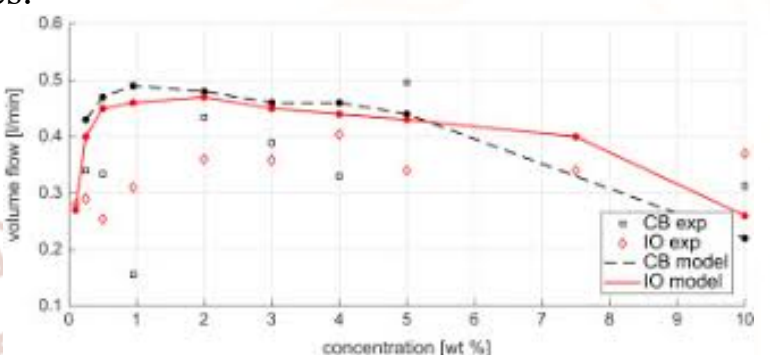

Figure 6: Time-average evaporation efficiency as a function of nanoparticle concentration for $\mathrm{CBNF}$ and

\subsection{Boiling Kinetics}

\section{IONF}

Making use of mass reduction curves from Fig. 4, it is possible to evaluate the volumetric flow of the steam produced in both nanofluids. The result is presented in Fig. 7 for different nanoparticle concentrations, where we also focus on concentrations not shown in Fig. 4. The flow rate exhibits complex non-monotonic behavior, initially reducing at the lowest mass fraction down to a minimum of approximately $0.5 \%$. This most probably occurs when the temperature probe, acting itself as a steam generation surface, becomes shielded by the nanoparticles, so that the evaporation driven solely by nanoparticles begins. The production rates further rise to maxima at $2 \%, 5 \%(\mathrm{CBNF})$ and $4 \%$ (IONF). We observed and described a similar behavior of the efficiencies for CBNF in Figs. 5-6, although the volume flow rate demonstrates greater sensitivity to concentration. Comparison of production rates for $\mathrm{CBNF}$ and IONF should be performed taking into account the difference in density and particle size between the nanofluids as IONPs, $<50$ times outnumbered relative to CBNPs, and generating steam at just slightly lower rate.

As seen in Fig. 7 the average production rate for both nanofluids is $0.4 \mathrm{l} / \mathrm{min}$ at the effective absorption area $1.210-3 \mathrm{~m} 2$. This amount of steam could be potentially utilized in a nano-turbine application, for example QuasiTurbine [29] (2 kW type), which
It is interesting to provide an order-of-magnitude estimate for the steam generation rate. This will furthermore depend on the nanofluid granulometry as the nanoparticles agglomerate when agitated in water due to thermal convection. This takes place at velocity $\mathrm{u}=\mathrm{g} \beta \Delta \mathrm{TcH} \approx 0.07 \mathrm{~m} / \mathrm{s}$, where $\Delta \mathrm{Tc}$ is the maximum temperature difference along the nanofluid column and $\mathrm{H}$ is the height of the boiling column. The nanoparticles cease to agglomerate when the adhesive van-der-Waals force, acting on agglomerate of size $\mathrm{da}, \mathrm{Fa}=\mathrm{Ada} / 24 \mathrm{~h} 2$ [30], becomes counter-balanced by the force of viscous retardation from the fluid $\mathrm{Fd}=$ $0.75 \pi \mu \mathrm{d} 2 \mathrm{vr} / \mathrm{h} 0$ [31], where the inter-particle relative velocity is proportional to the shear rate $\mathrm{vr} \approx \gamma \mathrm{da}$. Then the agglomerate size reads:

$$
d a=\sqrt{\frac{A}{18 \pi \mu \gamma h 0}}
$$

Where $\mathrm{ACB}=4.5 \cdot 10-20 \mathrm{~J}[32]$ and $\mathrm{AIO}=2.0 \cdot 10-20$ $\mathrm{J}$ [21] are the nanoparticle Hamaker constants (calculated in water following [30]), $\gamma \approx 40 \mathrm{~s}^{-1}$ is the shear rate (estimated using the aforementioned flow velocity and the inner diameter of the tube), $\mu=0.9$ $\mathrm{mPa} \cdot \mathrm{s}$ is dynamic viscosity and $\mathrm{h} 0 \approx 0.2 \mathrm{~nm}$ is the cut-off distance [30]. Equation (3) returns da, $\mathrm{CB}=$ $10.5 \mu \mathrm{m}$ and da,IO $=7.0 \mu \mathrm{m}$, being in satisfactory agreement with the results of optical microscopy (CBNP sample, Fig. S.2) where da,CB $7 \mu \mathrm{m}$ was detected. 
Following Mikic et al. [33], we define the minimum superheat that is required to generate steam shells around the nanoparticle agglomerates:

$$
\Delta T=\frac{4 \sigma T s}{\rho_{\vartheta} d_{a} r_{\rho \vartheta}}
$$

Where $\mathrm{Ts}=373 \mathrm{~K}$ is the saturation temperature, $\sigma=$ $60 \mathrm{mN} / \mathrm{m}$ is the surface tension, $\rho \mathrm{v}=0.59 \mathrm{~kg} / \mathrm{m} 3$ is the steam density and $\mathrm{rlv}=2.23 \cdot 106 \mathrm{~J} / \mathrm{kg}$. Using particle sizes, calculated with Equation (3), we obtain $\triangle \mathrm{TCB}=$ $6.5 \mathrm{~K}, \Delta \mathrm{TIO}=9.7 \mathrm{~K}$, which is again in agreement with the time-average experimental values from Table 1; estimation for IONF demonstrates greater discrepancies. The observed correspondence between the theoretical and the experimental superheat may probably support scenario of vapour shells formation, suggested by Neumann et al. [5].

Where $n b$ is the number density of the steam bubbles, estimated as reciprocal of the maximum steam bubble volume $\mathrm{Vb}, \max$.

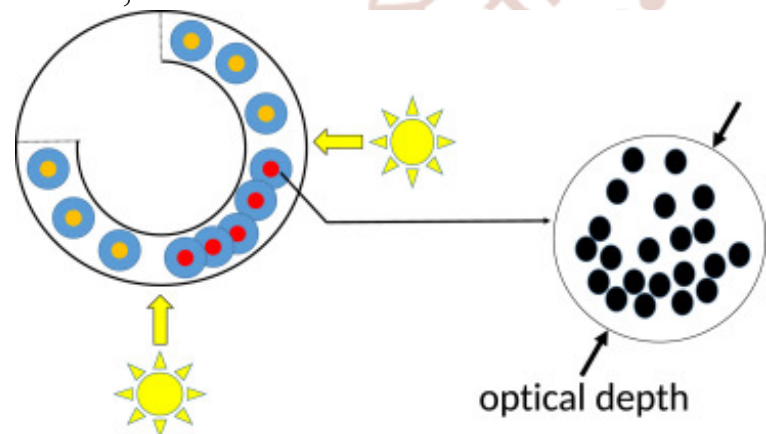

Figure 8: Schematic description of photothermal boiling. Steam bubbles incorporate nanoparticles. The hottest zone, irradiated with two lamps, is highlighted in red

We estimated the volumetric flow rate, also accounting for wet ability of the nanoparticle surface. For hydrophobic CBNPs the wetting angle was selected to be $\chi \mathrm{CB} 3 \pi / 4$, and for hydrophilic IONPs $\gamma \mathrm{IO}=0 . \quad$ The calculation results of the model are shown in Fig. (7) For different concentrations of nanoparticles. The theory agrees well with the experimental results both qualitatively and quantitatively, exhibiting two maxima at $1 \%$ and $2 \%$ for CBNF and IONF respectively. The most important discrepancies are apparently observed in the region of low concentrations, clearly demonstrating the effect of steam generation at the sensor probe. The calculation slightly overestimates the experimental results because the model does not account for a temporal delay due to nucleation of the primary steam bubble. Moreover, the surface Plasmon resonance was not considered for IONF. It is interesting to note that the bubble growth time $\tau \mathrm{g}(\sim 10-30 \mathrm{~ms})$ was of the same order as the average time, required for the nanoparticles to settle in the steam bubble of $d b, \max$, that was calculated assuming the Stokesian settling of the nanoparticles. Most of the nanoparticles were therefore located at the bottom of the maximum bubble. Taking into account density difference for CBNPs and IONPs, it is possible to assume the steam bubbles of IONF were less mechanically stable.

\section{CONCLUSION}

This paper reports experimental results on the photo thermal boiling of two different aqueous nanofluids, produced with carbon black and iron oxide nanoparticles. The "luminate" steam, generated in the fluids by the use of artificial radiation, was superheated stronger (up to $15.8 \mathrm{~K}$ ) than this would be expected for the conventional heating of water at the experimental heat flux. By taking a closer look at the granulometry of the produced nanofluids, we were able to provide an order-of-magnitude theoretical estimate of the superheat observed.

The iron oxide nanofluid demonstrated better thermal performance than the carbon black nanofluid, continuously increasing efficiency of the process with mass concentration (up to $75 \%$ at $10 \% \mathrm{wt}$ ) and more intense superheating the steam. The optimum concentration of $3 \% \mathrm{wt}$, on contrary, was detected for the carbon black nanofluid, with the maximum efficiency of $66 \%$. Inspecting condensate of the "luminate" steam, we observed significant amount of the iron oxide nanoparticles, which makes this nanofluid less attractive from the environmental viewpoint. The condensate from the carbon black nanofluid was free of the nanoparticles.

Adopting a simplistic heat balance method from the literature, it was possible to replicate steam production rate theoretically. Our model, which was based on the theoretical work by Dietzel and Poulikakos [36], demonstrated modest discrepancies relative to the experiment. We have also analyzed the kinetics of steam generation, up scaling the process for a potential use in a solar generator system for micro-CHP (up to $500 \mathrm{kWe}$ ). Following our estimates, the technology potentially becomes competitive with the photo-voltaic when an organic base fluid with lower latent heat is used instead of water. 


\section{REFERENCES}

1. T. Otanicar, P. Phelan, R. S. Prasher, G. Rosengarten, R. A. Taylor, Nanofluid-based direct absorption solar collector, Journal of Renewable and Sustainable Energy 2 (2010) 033102.

2. M. Karami, M. Akhavan-Bahabadi, S.Delfani, M.Raisee, Experimental investigation of $\mathrm{CuO}$ nanofluid- based direct absorption solar collector for residential applications, Renewable and Sustainable Energy Reviews 52 (2015) 793-801.

3. R. Taylor, P. Phelan, T. Otanicar, R. Adrian, R. S. Prasher, Nanofluid optical property characterization: Towards efficient direct absorption solar collectors, Nanoscale Research Letters 6 (2011) 1931-7573.

4. G. Ni, N. Miljkovic, H. Ghasemi, X. Huang, S. V. Boriskina, C.-T. Lin, J. Wang, Y. Xu, M. M. Rahman, T. J. Zhang, G. Chen, Volumetric solar heating of nanofluids for direct vapor generation, Nano Energy 17 (2015) 290-301.

5. O. Neumann, A. S. Urban, J. Day, S. Lal, P. Nordlander, N. J. Halas, Solar vapor generation enabled by nanoparticles, ACS Nano 7 (2013) 4249.

6. O. Neumann, C. Feronti, A. D. Neumann, A. Dong, K. Schell, B. Lu, E. Kim, M. Quinn, S. Thompson, N. Grady, P. Nordlander, M. Oden, N. J. Halas, Compact solar autoclave based on steam generation using broadband light-harvesting nanoparticles, PNAS 110 (2013) 11677-11681.

7. O. Neumann, A. D. Neumann, E. Silva, C. AyalaOrozco, S. Tian, P. Nordlander, N. J. Halas, Nanoparticle-mediated, light-induced phase separations, Nano Letters 15 (2015) 7880-7885.

8. O. Neumann, A. D. Neumann, S. Tian, C. Thibodeaux, S. Shubhankar, J. Mu"ller, E. Silva, A. Alabastri, S. W. Bishnoi, P. Nordlander, N. J. Halas, Combining solar steam processing and solar distillation for fully off-grid production of cellulosic bioethanol, ACS Energy Letters 2 (2017) 8-13.
9. A. Zeiny, H. Jin, G. Lin, P. Song, D. Wen, Solar evaporation via nanofluids: A comparative study, Renewable energy 122 (2018) 443-454.

10. H. Jin, G. Lin, L. Bai, A. Zeiny, D. Wen, Steam generation in a nanoparticle-based solar receiver, Nano Energy 28 (2016) 397-406.

11. M. Matsumoto, Surface Tension and Stability of a Nanobubble in Water: Molecular Simulation, Journal of Fluid Science and Technology 3 (2008) 922-929.

12. X. Wang, Y. He, G. Cheng, L. Shi, X. Liu, J. Zhu, Direct vapor generation through localized solar heating via carbon-nanotube nanofluid, Energy Conversion and Management 130 (2016) 176183.

13. M. M. Rahman, H. Younes, G. Ni, J. Y. Lu, A. Raza, T. J. Zhang, N. X. Fang, A. Al Ghaferi, Plasmonic nanofluids enhanced solar thermal transfer liquid, AIP Conference proceedings 1850 (2017) 110013.

14. X. Wang, Y. He, X. Liu, L. Shi, J. Zhu, Investigation of photothermal heating enabled by plasmonic nanofluids for direct solar steam generation, Solar Energy 157 (2017) 35-46.

15. Y. Fu, T. Mei, G. Wang, A. Guo, G. Dai, S. Wang, J. Wang, J. Li, X. Wang, Investigation on enhancing effects of $\mathrm{Au}$ nanoparticles on solar steam generation in graphene oxide nanofluids, Applied Thermal Engineering 114 (2017) 961968.

16. A. Guo, Y. Fu, G. Wang, X. Wang, Diameter effect of gold nanoparticles on photothermal conversion for solar steam generation, RCS Advances 7 (2017) 4815-4824.

17. H. Li, Y. He, Z. Liu, Y. Huang, B. Jiang, Synchronous steam generation and heat collection in a broadband $\mathrm{Ag} @ \mathrm{TiO} 2$ core-shell nanoparticlebased receiver, Applied Thermal Engineering 121 (2017) 617-627. 